\title{
Compressibility of microporous materials with CHA topology: 2. ALPO-34
}

\author{
Lara Leardini ${ }^{\mathrm{I}}$, Simona Quartieri ${ }^{\mathrm{I}}$, Annalisa Martucci ${ }^{\mathrm{II}}$, Maria Giovanna Vezzalini*,III and Vladimir Dmitriev $^{\mathrm{IV}}$ \\ I Dipartimento di Scienze della Terra, Viale Ferdinando Stagno d'Alcontres 31, 98166 Messina - S. Agata, Italy \\ II Dipartimento di Scienze della Terra, Sezione di Mineralogia, Petrografia e Geofisica, Via Saragat 1 32, 44100 Ferrara, Italy \\ III Dipartimento di Scienze della Terra, Largo Sant' Eufemia, 19, 41100 Modena, Italy \\ IV Swiss-Norwegian Beam Line at ESRF, BP220, 38043 Grenoble Cedex, France
}

In memoriam Professor Friedrich Liebau

Received November 9, 2011; accepted December 25, 2011

Published online: July 2, 2012

\section{ALPO-34 / CHA-framework type / Elastic behavior / $S R-X R P D$}

\begin{abstract}
The HP behavior of ALPO-34 as-synthesized was investigated by means of in-situ synchrotron X-ray powder diffraction, in the frame of a wider project aimed at understanding the role of the framework/extraframework content in the $P$-induced deformation mechanisms of natural and synthetic microporous materials with CHA framework topology. ALPO-34 compressibility under non-penetrating $P$ transmitting medium was determined up to $6.0 \mathrm{GPa}$ and upon decompression to $P_{\text {amb. After an initial large structure }}$ deformation at $P<0.4 \mathrm{GPa}$, a regular volume reduction was observed up to about $3 \mathrm{GPa}$. Above $3.1 \mathrm{GPa}$, an abrupt change in the behavior of all cell parameters was observed, accompanied by an evident decrease in compressibility. The isothermal Equation of State (EoS), refined with a II-order Birch-Murnaghan EoS from 0.4 to $3.1 \mathrm{GPa}$, yielded the following parameters: $V_{0}=755(1) \AA^{3}, K_{0}=54(3) \mathrm{GPa}$. No complete X-ray amorphization was achieved up to the highest investigated $P$ value. A complete reversibility of the unit cell parameters was observed upon $P$ release. The compressibility behavior of ALPO-34 was compared with that of the other CHA-type zeolites. The volume reduction observed for natural chabazite, and for SAPO-34 and ALPO-34 assynthesized, was $6.2 \%, 9.4 \%$, and $6.0 \%$, respectively. Notwithstanding the presence of morpholine molecules, as a structure directing agent, in the two as-synthesized phases, they exhibited significantly different compressibility. This can be interpreted as due to the octahedral coordination of part of the ALPO-34 framework aluminum, leading to a more rigid framework compared to that of SAPO-34, which contains only tetrahedral aluminum.
\end{abstract}

\section{Introduction}

Microporous materials are of great importance, both from the geological point of view (Bish \& Ming, 2001), and in

\footnotetext{
* Correspondence author

(e-mail: mariagiovanna.vezzalini@unimore.it)
}

many fields of applied chemistry, such as ion exchange, molecular sieving, and catalysis (Bish \& Ming, 2001; Corma, 1997). In addition to natural and synthetic aluminosilicatic zeolites, microporous aluminophosphates $\left(\mathrm{AlPO}_{4}\right)$ and silicoaluminophosphates (SAPO) molecular sieves have also been extensively studied in view of their use in industrial processes, including catalysis and gas separation (Leardini et al., 2010 and reference there cited).

In recent years, numerous studies on the high-pressure (HP) stability of zeolites have been performed (Fois et al., 2008; Ori et al., 2008a; Ori et al., 2008b; Gatta, 2008; Arletti et al., 2010; Gatta, 2010; Arletti et al., 2011; Quartieri et al., 2011). In these investigations, the crucial role of the extra-framework content on the zeolite response in terms of deformation mechanisms and compressibility values - emerged clearly. On the contrary, the effect of framework composition on compressibility and HP stability of microporous materials has not yet been systematically investigated.

Recently, the HP behavior of selected natural and synthetic microporous materials with CHA framework topology (natural chabazite and as-synthesized SAPO-34) was investigated by means of in-situ synchrotron X-ray powder diffraction and silicone oil as non-penetrating $P$-transmitting medium, with the aim of understanding the role of the framework/extraframework composition in the $P$-induced deformation mechanisms (Leardini et al., 2010). The results indicate that chabazite is definitely less compressible than SAPO-34. This was mainly ascribed to the more complex and bulky extraframework content of the natural sample. No complete structural refinements of the powder patterns were performed, but the compressibility of these materials with CHA topology was interpreted on the basis of the deformation mechanism observed in the structurally related zeolite levyne (Gatta et al., 2005).

The aim of this work is to extend investigation into the compressibility of zeolites with CHA topology to ALPO34 as-synthesized, in order to understand the influence of the extra-framework, and in particular, of the framework composition on stability, degree of compressibility, and pore deformation under pressure. The compressibility of 
ALPO-34 as-synthesized is directly compared to that of SAPO-34, since both materials contain morpholine as a structure directing agent. The presence of molecular complexes or aggregates of templating molecules is expected to play a decisive role in the response of materials to compression. Furthermore, a comparison between these two microporous materials will enable an evaluation of the effect of the different framework structure, which in ALPO34 is characterized by the unusual presence of octahedrally coordinated $\mathrm{Al}$ atoms.

\section{Experimental}

\section{Sample structure and composition}

The CHA-type framework structure can be described as an ABC sequence of double 6-rings of tetrahedra linked together by single 4-rings ("joint unit") (Dent \& Smith, 1958; Smith et al., 1963; Smith et al., 1964; Mazzi et al., 1983; Engelhardt \& Michel, 1987). The resulting framework is characterized by a three dimensional channel system perpendicular to [111] direction (in the rhombohedral setting) and confined by 8 -membered rings (Fig. 1). The channel intersection gives rise to the so-called chabazitecage, which may host extraframework species.

In ALPO-34 synthesized in HF medium with morpholine as template, the asymmetric unit contains three tetra-

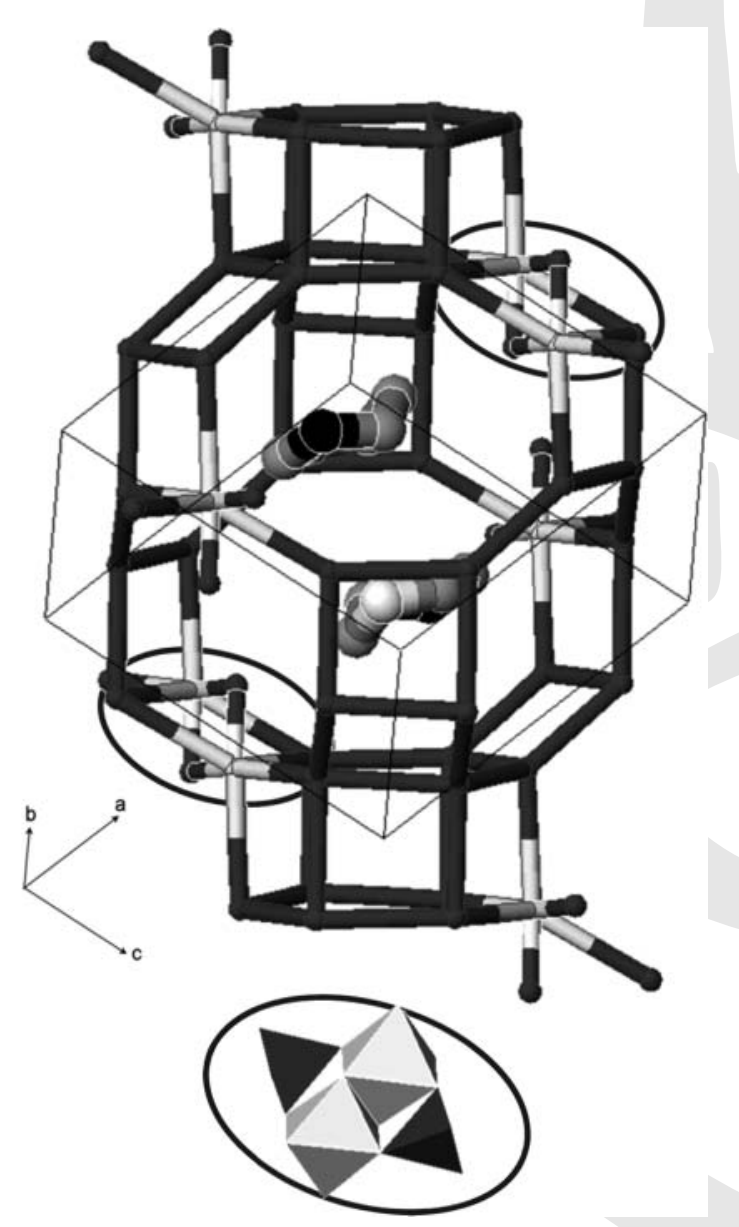

Fig. 1. ALPO-34 as-synthesized structure. Solid lines represent T-T linkages. The inset shows the "joint-unit" containing two octahedrally coordinated $\mathrm{Al}$ atoms. Two morpholine molecules reside in the chabazite cage. hedral $P$, two tetrahedral $\mathrm{Al}$, and a further $\mathrm{Al}$ octahedrally coordinated by four $\mathrm{O}$ and two $\mathrm{F}$ atoms. Bridging fluorine atoms draw the $\mathrm{Al}$ atoms closer together, causing a distortion of the framework and a reduction of the typical chabazite topological symmetry (rhombohedral $R \overline{3} m$, Dent \& Smith, 1958; Smith et al., 1963) to the real symmetry $P \overline{1}$ of AlPO-34.

Morpholine $\left(\mathrm{C}_{4} \mathrm{H}_{9} \mathrm{NO}\right)$ is a heterocyclic amine consisting of a six membered ring in a chair conformation. A pair of hydrogen atoms bind each carbon atom, whereas only one hydrogen is bonded to nitrogen. In ALPO-34, the template is present in its protonic form (morpholinium $\mathrm{C}_{4} \mathrm{H}_{10} \mathrm{NO}^{+}$), where nitrogen is also bonded to a pair of hydrogen atoms. The positive charge is counter-balanced by the $\mathrm{F}^{-}$ions. FTIR and Raman spectroscopy shows that the $\mathrm{NH}_{2}$ group of morpholinium is $\mathrm{H}$-bonded to the zeolitic framework by rather weak interactions (Marchese et al. 1999). Harding \& Kariuki (1994) found the template in two fully occupied sites in the chabazite-cage (Fig. 1).

The as-synthesized ALPO-34 studied here was prepared by Marchese et al. (1999) in the following composition: $\mathrm{Al}_{6}\left(\mathrm{PO}_{4}\right)_{6} 2 \mathrm{~F}^{-} \cdot 2\left(\mathrm{C}_{4} \mathrm{H}_{8} \mathrm{NOH}_{2}\right)^{+}$.

As previously stated, in this study the elastic behavior of ALPO-34 is directly compared with that of SAPO-34 (s.g. $R \overline{3} ; \quad \mathrm{Si}_{0.9} \mathrm{Al}_{6.0} \mathrm{P}_{5.1} \mathrm{O}_{24} \cdot 0.9\left(\mathrm{C}_{4} \mathrm{H}_{8} \mathrm{NOH}_{2}\right)^{+} \cdot 1.1\left(\mathrm{C}_{4} \mathrm{H}_{8} \mathrm{NOH}\right)$ - $2.7 \mathrm{H}_{2} \mathrm{O}$ ), the other synthetic CHA-type phase already studied under HP (Leardini et al. 2010). The two phases are characterized by different framework composition: $\mathrm{Si}, \mathrm{Al}$, $P$ in SAPO-34, and $\mathrm{Al}$ and $P$ in ALPO-34. Si atoms substitute only for phosphorous atoms in SAPO-34, thus causing a negatively charged framework, while the ALPO34 framework is neutral. Moreover, two fluorine atoms/ u.c. are present in ALPO-34, but not in SAPO-34. As regards the extraframework, both phases contain two morpholine/morpholinium molecules/u.c. In SAPO-34, 2.7 water molecules are also present (Martucci et al., 2003; Marchese et al., 1999). On the contrary, both FTIR and TG analyses (Marchese et al. 1999) rule out the presence of water in ALPO-34.

In SAPO- 34 about $2 / 3$ of the organic molecules are protonated whereas the residual $1 / 3$ are neutral morpholine (Martucci et al. 2003). Single crystal X-ray analysis and FTIR spectroscopy indicated the occurrence of water-morpholinium/morpholine chains embedded in the zeolitic pores (Martucci et al. 2003).

\section{X-ray powder diffraction at room pressure}

A preliminary X-ray powder diffraction characterization at room pressure $\left(P_{\mathrm{amb}}\right)$ was performed at the Centro Interdipartimentale Grandi Strumenti (CIGS) of the University of Modena and Reggio Emilia, using a Panalytical X'Pert PRO diffractometer $\left(\theta / \theta\right.$ geometry, $\mathrm{Cu} K_{\alpha}$ radiation $)$ equipped with an X-celerator detector. A parabolic X-ray mirror was mounted on the incident beam to focus the beam on the sample. The powder was loaded in a boron capillary and spun under the X-ray beam. Data were collected in the $2 \theta$ range $3-120^{\circ}$ with steps of $0.02^{\circ}$ and at $900 \mathrm{~s}$ per step speed. 


\section{Synchrotron X-ray powder diffraction experiments under high pressure}

The high pressure X-ray powder diffraction (XRPD) experiments were performed at the SNBL1 (BM01a) beamline at ESRF (European Synchrotron Facility) with a fixed wavelength of $0.71 \AA$, using a modified Merril-Basset DAC (Miletich et al., 2000) and silicone oil as the nonpenetrating $P$-transmitting medium (Ragan et al. 1996, Angel et al. 2007). The pressure was measured using the ruby fluorescence method (Forman et al., 1972) on the non linear hydrostatic pressure scale (Mao et al., 1986). The estimated error in the pressure values is $0.1 \mathrm{GPa}$. A MAR345 detector (pixel dimension $150 \mu \mathrm{m}$ ) was used at a fixed distance of $230 \mathrm{~mm}$ from the sample; the exposure time was $300 \mathrm{~s}$ for all pressure points. These experimental conditions were chosen to allow comparison with data collected for other CHA-type materials studied previously by our group (Leardini et al. 2010). The experiments were performed from $P_{\text {amb }}$ up to $6.6 \mathrm{GPa}$. The $\Delta P$ increments were of $0.2-0.8 \mathrm{GPa}$. Other patterns were measured while decompressing the sample at $5.6 \mathrm{GPa}$ and room conditions, as reported in Fig. 2 and Table 3. One-dimensional diffraction patterns were obtained in the $2 \theta$ range $0-47^{\circ}$ by integrating the two-dimensional images with the program FIT2D (Hammersley et al., 1996). A total of 18 spectra were collected.

\section{Refinements strategy}

Rietveld structure refinement of data collected at $P_{\text {amb }}$ was performed in the $P \overline{1}$ space group, using the initial structural model proposed by Harding \& Kariuki (1994) for an ALPO-34 sample synthesized with morpholine as structure directing agent. The background curve was fitted using a Chebyschev polynomial with 27 variable coefficients. Profiles were modeled using a pseudo-Voight peak-shape function (Thompson et al., 1987) including correction for axial divergence asymmetry (Finger et al., 1994) for low angle peaks. Soft constraints were imposed on $\mathrm{T}-\mathrm{O}$, $\mathrm{O}-\mathrm{O}, \mathrm{C}-\mathrm{C}, \mathrm{C}-\mathrm{O}$, and $\mathrm{C}-\mathrm{N}$ bond distances, and then released in the last stages of the refinement. Hydrogen atoms were not considered in the structure refinement due to their low scattering factors. In the final cycles, the refined structural parameters were the following: fractional coordinates for all atoms, occupancy factors for extraframework cations, and isotropic displacement factors for all atoms. Refinement of site occupancy of morpholine converged to 1.00 , which is the maximum expected value on the basis of the chemical composition of the sample under study. Except for small discrepancies at low $\sin \theta / \lambda$, caused by asymmetric line profiles, the Rietveld fit was satisfactory, even for low Bragg intensities.

The structural model refined for data at ambient conditions was then used for the analysis of the HP patterns. Due to a number of uncontrollable effects, including the poor statistics of the diffraction data and the possible development of preferred orientations (Arletti et al., 2003; Gatta et al., 2006), the peak intensities of these spectra were not reliable and only the unit cell parameters were extracted from the powder patterns.

The refinements of the cell parameters were carried out in the $3-36^{\circ} 2 \theta$ range by means of the Rietveld method up to $3.1 \mathrm{GPa}$. Above this pressure, any attempt to refine the unit cell parameters using the Rietveld method failed and no convergence was achieved. A careful examination of the powder diffraction patterns showed that in the 3.13.9 GPa range, a strong shift of $(00 l)$ reflections towards higher $2 \theta$ occurred. At the same time, a slight variation of (h00) and $(0 k 0)$ reflection positions was also observed. These observations suggest a remarkable structural rearrangement upon compression. The powder pattern at 3.9 GPa was then indexed using the EXPO 2009 program (Altomare et al., 2009) which suggested the following triclinic cell: $a=9.143(3), \quad b=8.987(3), \quad c=8.782(3), \quad \alpha=$ 87.37(3), $\beta=100.90(2), \gamma=91.99(2), V=707.5(4)$. The refinement of the unit cell parameters was then performed using LeBail method of GSAS package (Larson \& Von Dreele, 1996) with the EXPGUI interface (Toby, 2001). No deviation from the $P \overline{1}$ symmetry was observed and this space group was maintained until the highest refined pressure value $(6.0 \mathrm{GPa})$. The scale factor and $2 \theta$-zero shift were accurately refined in all patterns. The background curves were fitted by a Chebyschev polinomial function with 18 coefficients. The pseudo-Voight profile function proposed by (Thompson et al., 1987) was used with refined Gaussian (GW) and Lorentzian (LX) terms and cut-off of the peak intensities was applied.

\section{Results}

\section{AIPO-34 structure at ambient conditions}

The structural features determined for the study sample at $P_{\text {amb }}$ are similar to those reported by Harding \& Kariuki (1994) for AlPO-34 as-synthesized. Table 1 summarizes the refinement details; Table 2 reports atomic coordinates, occupancy factors and isotropic thermal parameters.

Table 1. Unit cell and XRPD refinement parameters for ALPO-34 as synthesized at $P_{\text {amb }}$.

\begin{tabular}{ll}
\hline Space Group & $P \overline{1}$ \\
\hline$a(\AA)$ & $9.3337(6)$ \\
$b(\AA)$ & $9.1768(6)$ \\
$c(\AA)$ & $9.1629(6)$ \\
$\alpha\left(^{\circ}\right)$ & $88.423(5)$ \\
$\beta\left(^{\circ}\right)$ & $102.519(5)$ \\
$\gamma\left({ }^{\circ}\right)$ & $93.654(5)$ \\
$V\left(\AA^{3}\right)$ & $764.55(8)$ \\
$2 \theta$ range & $6-120$ \\
$R_{w p}(\%)$ & 5.91 \\
$R_{p}(\%)$ & 3.93 \\
$R_{F}^{2}(\%)$ & 13.91 \\
$N$ of reflections & 6689 \\
$N_{\text {obs }}$ & 4780 \\
$N_{\text {var }}$ & 121 \\
\hline
\end{tabular}


Table 2. Refined atomic positions, occupancy factors and displacement parameters $\left(\AA^{2}\right)$ of ALPO-34 as synthesized at $P_{\text {amb. }}$.

\begin{tabular}{llllll}
\hline & $x / a$ & $y / b$ & $z / c$ & Frac & UISO \\
\hline A11 & $0.9210(7)$ & $0.0597(7)$ & $0.3771(7)$ & 1 & $0.016(1)$ \\
A12 & $0.0714(8)$ & $0.3426(8)$ & $0.8644(8)$ & 1 & $0.016(1)$ \\
A13 & $0.3480(8)$ & $0.8785(8)$ & $0.1076(9)$ & 1 & $0.016(1)$ \\
P1 & $0.8989(8)$ & $0.3128(8)$ & $0.1164(7)$ & 1 & $0.016(1)$ \\
P2 & $0.3209(8)$ & $0.1188(8)$ & $0.8726(8)$ & 1 & $0.016(1)$ \\
P3 & $0.1471(8)$ & $0.8564(8)$ & $0.3306(8)$ & 1 & $0.016(1)$ \\
F1 & $0.9092(9)$ & $0.8800(7)$ & $0.4848(10)$ & 1 & $0.020(2)$ \\
O1 & $0.7102(8)$ & $0.1209(12)$ & $0.7258(9)$ & 1 & $0.020(2)$ \\
O2 & $0.6642(15)$ & $0.9462(8)$ & $0.9672(8)$ & 1 & $0.020(2)$ \\
O3 & $0.7766(9)$ & $0.7522(10)$ & $0.1451(11)$ & 1 & $0.020(2)$ \\
O4 & $0.0265(11)$ & $0.3012(15)$ & $0.0367(9)$ & 1 & $0.020(2)$ \\
O5 & $0.0690(10)$ & $0.7633(8)$ & $0.7312(7)$ & 1 & $0.020(2)$ \\
O6 & $0.9558(10)$ & $0.0238(10)$ & $0.7296(13)$ & 1 & $0.020(2)$ \\
O7 & $0.9281(10)$ & $0.2926(8)$ & $0.7234(11)$ & 1 & $0.020(2)$ \\
O8 & $0.5273(6)$ & $0.8311(11)$ & $0.1481(12)$ & 1 & $0.020(2)$ \\
O9 & $0.8111(12)$ & $0.1437(10)$ & $0.4978(7)$ & 1 & $0.020(2)$ \\
O10 & $0.1199(11)$ & $0.5261(5)$ & $0.8570(13)$ & 1 & $0.020(2)$ \\
O11 & $0.2489(8)$ & $0.0018(8)$ & $0.7613(8)$ & 1 & $0.020(2)$ \\
O12 & $0.2408(10)$ & $0.7575(10)$ & $0.9835(11)$ & 1 & $0.020(2)$ \\
C1 & $0.523(3)$ & $0.547(3)$ & $0.736(4)$ & $1.000(5)$ & $0.039(5)$ \\
C2 & $0.408(3)$ & $0.651(4)$ & $0.652(4)$ & $1.000(5)$ & $0.039(5)$ \\
C3 & $0.601(4)$ & $0.770(3)$ & $0.541(4)$ & $1.000(5)$ & $0.039(5)$ \\
C4 & $0.661(4)$ & $0.615(3)$ & $0.562(3)$ & $1.000(5)$ & $0.039(5)$ \\
N1 & $0.508(3)$ & $0.782(3)$ & $0.652(3)$ & $1.000(5)$ & $0.039(5)$ \\
Om & $0.669(3)$ & $0.574(3)$ & $0.715(2)$ & $1.000(5)$ & $0.039(5)$ \\
\hline & & & & &
\end{tabular}

In particular, one third of the aluminum atoms were found in octahedral coordination and two fully occupied sites of morpholine were found in the unit cell (Fig. 1). The two organic molecules are related by the inversion center in the middle of the chabazite cage. The large distance between them prevents their interaction via H-bonding. In contrast, the bond distances of the $\mathrm{N}$ atoms of the morpholine molecules with the framework oxygen atoms O2 and O9 (3.30(3) and 3.11(3), respectively) suggest the existence of $\mathrm{H}$-bonding interactions.

\section{AlPO-34 at high pressure: unit-cell parameters}

Figure 2 shows selected powder patterns of ALPO-34 as a function of pressure; the peak intensities decrease and the peak profiles become broader with increasing pressure. Notwithstanding this, complete X-ray amorphization is not achieved up to the highest investigated pressure.

Above $3.1 \mathrm{GPa}$ an abrupt change in the powder patterns is observed. However, since all the observed peaks are consistent with the $P \overline{1}$ s.g., a $P$-induced change of the original symmetry can be excluded. The two patterns collected upon decompression down to room conditions demonstrate that the $P$-induced effects on the ALPO-34 structure are reversible. All the features of the $P_{\text {amb }}$ pattern and the unit cell parameters were well recovered upon $P$ release.

Figure 3 and Table 3 report the values of the cell parameters as a function of pressure in the range $P_{\mathrm{amb}}-6.0 \mathrm{GPa}$

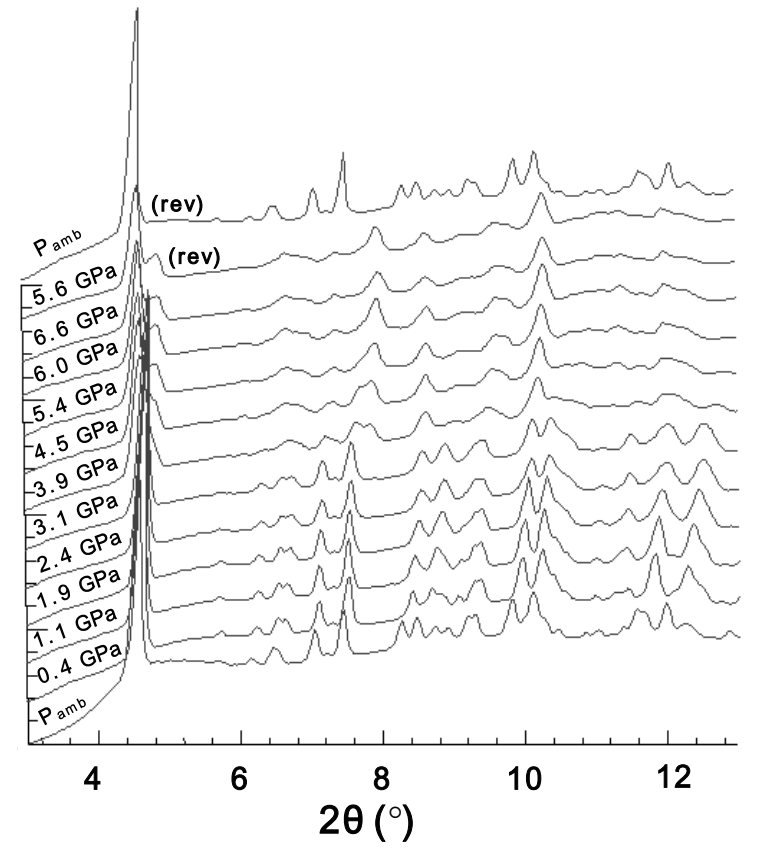

Fig. 2. Selected integrated powder patterns of ALPO-34 as-synthesized as a function of pressure, reported in the $2 \theta$ range $2-14^{\circ}$. The powder patterns at the top of the figure were collected during decompression.

and upon decompression. Two distinct trends are visible at pressure values lower and higher than $3.1 \mathrm{GPa}$.

In the first $P$-range, the cell axes decrease regularly while the cell angles increase, this opposite behavior being ascribable to mechanical causes linked to the shape of the pseudo-rhombohedric chabazite cage. The unit cell volume decrease is $6 \%$, similarly to that observed in natural cha-

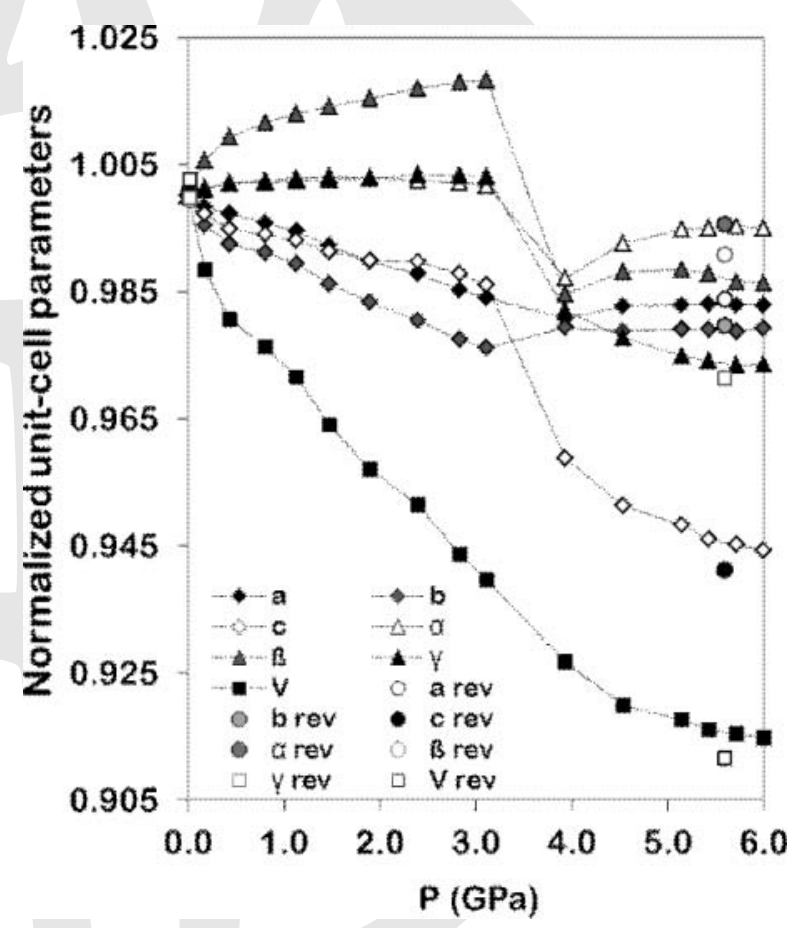

Fig. 3. Variation of ALPO-34 as-synthesized lattice parameters as a function of pressure. The errors associated with the cell parameters are smaller than the symbols used. 
Table 3. Unit-cell parameters of ALPO-34 as synthesized at the investigated pressures.

\begin{tabular}{llllllll}
\hline$P(\mathrm{GPa})$ & $a(\AA)$ & $b(\AA)$ & $c(\AA)$ & $\alpha\left(^{\circ}\right)$ & $\beta\left(^{\circ}\right)$ & $\gamma\left({ }^{\circ}\right)$ & $V\left(\AA^{3}\right)$ \\
\hline$P_{\text {amb }}$ & $9.322(3)$ & $9.177(3)$ & $9.160(2)$ & $88.51(2)$ & $102.48(2)$ & $93.67(2)$ & $763.4(4)$ \\
0.2 & $9.305(3)$ & $9.134(3)$ & $9.134(2)$ & $88.61(2)$ & $103.05(2)$ & $93.79(2)$ & $754.6(4)$ \\
0.4 & $9.296(3)$ & $9.108(3)$ & $9.112(2)$ & $88.70(2)$ & $103.44(2)$ & $93.85(2)$ & $748.6(4)$ \\
0.8 & $9.282(3)$ & $9.095(3)$ & $9.105(2)$ & $88.73(2)$ & $103.66(2)$ & $93.86(2)$ & $745.3(4)$ \\
1.1 & $9.270(3)$ & $9.080(3)$ & $9.096(2)$ & $88.76(2)$ & $103.81(2)$ & $93.90(2)$ & $741.7(4)$ \\
1.5 & $9.249(3)$ & $9.050(3)$ & $9.080(2)$ & $88.78(2)$ & $103.92(2)$ & $93.91(2)$ & $736.0(4)$ \\
1.9 & $9.226(3)$ & $9.024(3)$ & $9.067(2)$ & $88.77(2)$ & $104.06(2)$ & $93.93(2)$ & $730.6(4)$ \\
2.4 & $9.208(3)$ & $8.997(3)$ & $9.066(2)$ & $88.73(3)$ & $104.22(2)$ & $93.98(2)$ & $726.3(4)$ \\
2.8 & $9.184(3)$ & $8.969(3)$ & $9.048(3)$ & $88.70(3)$ & $104.32(3)$ & $93.98(2)$ & $720.4(5)$ \\
3.1 & $9.172(3)$ & $8.959(3)$ & $9.032(3)$ & $88.67(3)$ & $104.36(2)$ & $93.95(2)$ & $717.3(4)$ \\
3.9 & $9.143(3)$ & $8.987(3)$ & $8.782(3)$ & $87.37(3)$ & $100.90(2)$ & $91.99(2)$ & $707.5(4)$ \\
4.5 & $9.160(6)$ & $8.981(3)$ & $8.713(4)$ & $87.86(4)$ & $101.26(4)$ & $91.58(5)$ & $702.3(6)$ \\
5.2 & $9.162(4)$ & $8.984(3)$ & $8.685(4)$ & $88.06(4)$ & $101.29(4)$ & $91.32(5)$ & $700.5(5)$ \\
5.4 & $9.163(4)$ & $8.984(3)$ & $8.665(3)$ & $88.07(4)$ & $101.22(4)$ & $91.24(5)$ & $699.3(5)$ \\
5.7 & $9.162(3)$ & $8.982(3)$ & $8.658(3)$ & $88.10(4)$ & $101.09(3)$ & $91.18(5)$ & $698.8(5)$ \\
6.0 & $9.162(4)$ & $8.986(4)$ & $8.649(4)$ & $88.07(4)$ & $101.08(4)$ & $91.20(5)$ & $698.3(5)$ \\
5.6 (rev) & $9.170(6)$ & $8.990(5)$ & $8.621(4)$ & $88.12(4)$ & $101.54(5)$ & $90.98(5)$ & $695.9(7)$ \\
$P_{\text {amb }}(\mathrm{rev})$ & $9.331(2)$ & $9.187(2)$ & $9.162(2)$ & $88.48(2)$ & $102.42(2)$ & $93.64(2)$ & $765.4(2)$ \\
\hline
\end{tabular}

bazite from Vallerano and lower than that of SAPO-34. The compressibility is not completely isotropic: the unit cell parameters variation are $-1.6 \%,-2.4 \%$, and $-1.4 \%$ for $a, b$, and $c$, respectively, while the cell angles $\alpha, \beta$, and $\gamma$ increase by $0.2 \%, 1.8 \%$, and $0.3 \%$, respectively.

In the second $P$-range, and in particular between 3.1 and $3.9 \mathrm{GPa}$, an abrupt decrease of the $c$ axis and of all the cell angles is observed, while $a$ decreases regularly and $b$ slightly increases. The $c$ axis and $\gamma$ continue to contract above $3.9 \mathrm{GPa}$, while the other parameters slightly increase or remain almost constant.

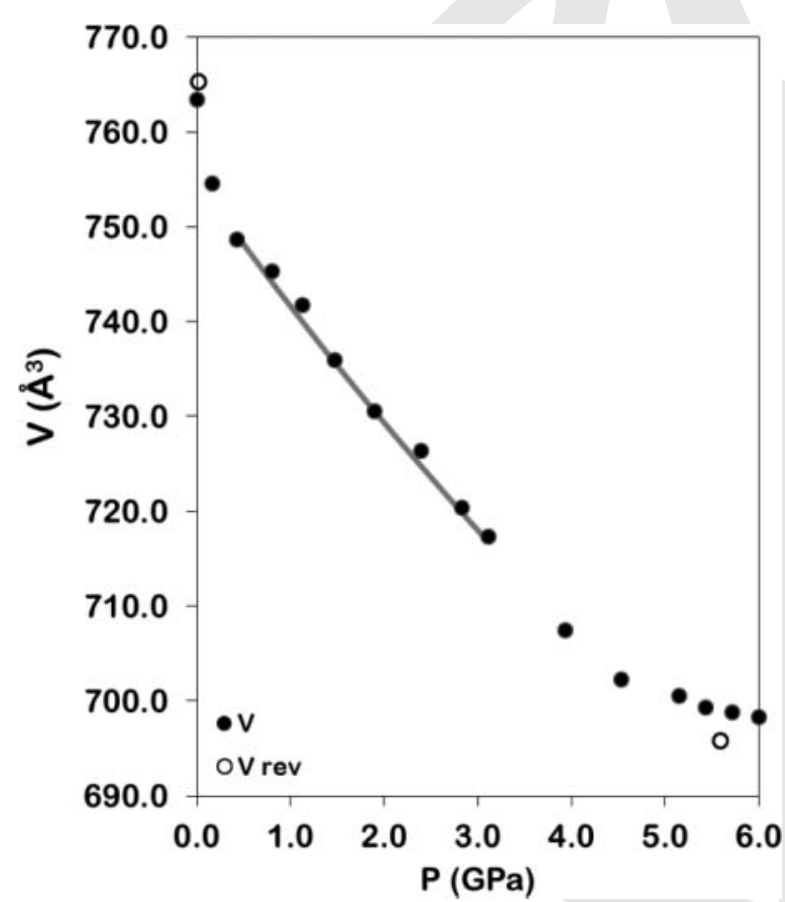

Fig. 4. Unit cell volume $v s$. pressure. The grey line represents the fit of the unit-cell volume vs. $P$ obtained using a second order BirchMurnaghan EoS between 0.4 and $3.1 \mathrm{GPa}$.
Concerning the $P$-induced unit cell volume variation, in Fig. 3 it can be seen that after an initial marked structure deformation in the very low- $P$ regime $\left(P_{\mathrm{amb}}-0.4 \mathrm{GPa}\right)$, regular volume reduction occurs up to $3.1 \mathrm{GPa}$. After this $P$ value, the abrupt change of all cell parameters does not correspond to a similar abrupt change in the cell volume, but, on the contrary, to a regular volume decrease. A successive compressibility decrease is observed above $4.5 \mathrm{GPa}$, possibly related to the hindrance of the extraframework morpholine molecules. We cannot, however, exclude an influence of the not completely hydrostatic behavior of silicone oil in this $P$ regime (Angel et al. (2007). The overall ALPO-34 volume decrease is of $8.5 \%$.

Due to these discontinuities in the elastic evolution of unit cell parameters, the bulk modulus was calculated in the $P$-range from 0.4 to $3.1 \mathrm{GPa}$ using a second-order Birch-Murnaghan Equation of State (II-BM-EoS; Birch, 1947). The calculation of the bulk modulus performed with the data weighted by the uncertainties in $P$ and $V$ gave the following parameters: $V_{0}=755(1) \AA^{3}$, $K_{0}=54(3) \mathrm{GPa}$ (Fig. 4). The refined linear axial bulk moduli are: $K_{0}(a)=58(3) ; K_{0}(b)=44(2) ; K_{0}(c)=97(7)$ for the $a, b$, and $c$-axis, respectively.

Tentative structural interpretation of ALPO-34 elastic behavior

Notwistanding the quality of the HP powder patterns did not allow complete structural refinement to be performed, we can tentatively interpret the elastic behavior of ALPO34 by comparing it with that of the structurally-related zeolite levyne (hexagonal, space group $R \overline{3} m, a_{\mathrm{H}} \approx$ $13.338 \AA, c_{\mathrm{H}} \approx 23.014 \AA$, Merlino et al. 1975). Its framework structure is characterized by layers of double and single six membered rings of tetrahedra connected through 
single 4-rings. Double-six ring layers alternate with single ring layers along $c$ direction resulting in an AABCCABBCAA stacking sequence. Levyne compressibility was studied by single-crystal XRD, and its deformation mechanism was elucidated through complete structural refinements in the hexagonal setting (Gatta et al. 2005). Hence, to allow the comparison with levyne, we have converted ALPO-34 triclinic parameters into the pseudo-hexagonal ones (see Fig. A of Appendix).

Also in this setting, an abrupt slope change in the evolution of the unit-cell parameters is observed above 3.1 GPa (see Fig. B Appendix 1): $c_{\mathrm{H}}$ parameter suddenly increases by about $4 \%$, whereas the three pseudo-hexagonal $a_{H}$ parameters decrease. This abrupt increase of $c_{H}$ could be interpreted as due to the increase of the thickness of the D6R, similarly to that observed in levyne at $P \sim 0.4$ $\mathrm{GPa}$. The different pressure at which this polyhedral tilting mechanism occurs in ALPO-34 and the higher extent of the $c_{H}$ expansion with respect to levyne, are probably due to two factors: i) the presence, in ALPO-34, of two Alcentered octahedra in the double six-rings and ii) to the $\mathrm{H}$ bonding interactions of morpholine molecules with the framework oxygen atoms: when the compression induces too short H-bond distances, a rearrangement of the morpholine molecules possibly occurs inducing a change in the compression mechanisms (increasing the pseudo hexagonal $c_{\mathrm{H}}$ parameter and decreasing the $a_{\mathrm{H}}$ parameters).

It is also interesting to observe that the thickness of the D6R in levyne at ambient pressure $(4.998(3) \AA)$ is very similar to that reported for SAPO-34 by Martucci et al. (2003) (4.97 $\AA$ ). In the case of ALPO-34, three different thickness values must be considered, since the double sixrings are corrugated. In particular, at $P_{\mathrm{amb}}$ the highest thickness of the D6R (O10-O9 = 5.17(1) $\AA$ ), corresponding to the inter-tetrahedral angle O10-O7-O9 $\left(150.4(6)^{\circ}\right)$, is higher than that reported for levyne in its maximum expanded configuration (at about $1 \mathrm{GPa}$ ) (Gatta et al. 2005). This suggests that no further increase of ALPO-34 D6R thickness is possible and justifies the initial decrease of $c_{H}$ upon compression up to $3.1 \mathrm{GPa}$. At this pressure, a possible change in the compression mechanism could be induced by the extraframework morpholine molecules or by other effects non detectable without detailed structural refinements.

\section{Discussion and concluding remarks}

Three porous materials with CHA framework type were studied under HP using silicon oil (s.o.) as non-penetrating $P$-transmitting medium: natural chabazite from Vallerano (Leardini et al., 2010), SAPO-34 as-synthesized (Leardini et al. 2010), and ALPO-34 as-synthesized (this work).

The common aspects of the response to pressure of these materials are: i) the absence of complete amorphization up to the highest applied $P$; ii) the absence of phase transitions; and iii) the more or less complete reversibility of the $P$-induced modifications of the cell parameters and of the XRPD pattern intensities. These findings suggest that CHA topology undergoes only displacive modifications.

The different compressibility of the three materials can be evaluated by comparing the volume decrease of the three phases in the same $P$-range, specifically from $P_{\text {amb }}$ to $4.5 \mathrm{GPa}$ (maximum pressure at which $\mathrm{SAPO}-34$ cell parameters were measured). The corresponding $\Delta V \%$ (7.7, 11.5, and 8.0 for chabazite (Leardini et al., 2010), SAPO34 (Leardini et al., 2010), and ALPO-34, respectively) clearly indicate that the less compressible phase is the natural chabazite, which is characterized by an alumino-silicatic framework and by the most complex extraframework content, characterized by several partially occupied cationic and water sites.

The comparison of the compressibility of the two synthetic phases gives an unexpected result on the basis of their extraframework contents: both materials contain morpholine as template, but ALPO-34 is almost anhydrous, while SAPO-34 contains about 3 water molecules. As largely discussed in literature (Fois et al., 2008; Ori et al., 2008a; Ori et al., 2008b; Gatta, 2008; Arletti et al., 2010; Gatta, 2010; Arletti et al., 2011; Quartieri et al., 2011) and specifically found for natural chabazite (Leardini et al. 2010), the extraframework water molecules largely contribute to sustaining the zeolite framework. This is even more important if we consider that water molecules in SAPO-34 form molecular chains with morpholine, passing through adjacent $\mathrm{CHA}$ cages, making it logical to expect a higher compressibility for the anhydrous ALPO-34.

The opposite observed behavior indicates that, in this case, zeolite compressibility is predominantly controlled by the framework features, and in particular by the presence of the two Al octahedra sharing an edge in the 4membered rings ("joint unit") which connect different double-six rings (Fig. 1). As a consequence, ALPO-34 framework is, as a whole, more rigid than SAPO-34.

The results of this work highlight the interest for future studies of the high pressure behavior of microporous mixed octahedral-tetrahedral frameworks (Krivovichev, 2005; Chukanov \& Pekov, 2005).

\section{Appendix}

Figure A shows the relationship between the triclinic pseudo-rhombohedric parameters of ALPO-34 $\left(a_{T}, b_{T}\right.$ and $\left.c_{T}\right)$ and the pseudo-hexagonal ones $\left(a 1_{H}, a 2_{H}\right.$ and $a 3_{H}$, with $\left.a 1_{H}>a 2_{H}>a 3_{H}\right)$.

The conversion to the pseudo-hexagonal setting was performed graphically, by drawing the framework structure and measuring the distances between an atom placed in the origin of the cell and the crystallographically equivalent ones, related by the three pseudo-hexagonal axes.

In Fig. B the trend of the normalized pseudo-hexagonal unit cell parameters is shown as a function of pressure.

Three different elastic regimes are observed:

i) $0<P<0.5 \mathrm{GPa}: c_{H}$ parameter decreases by about $2 \%$ followed by the decrease of both $a 2_{H}$ and $a 3_{H}$ parameters. On the contrary, $a 1_{H}$ parameter, which is the longest one, increases by about $0.5 \%$. 

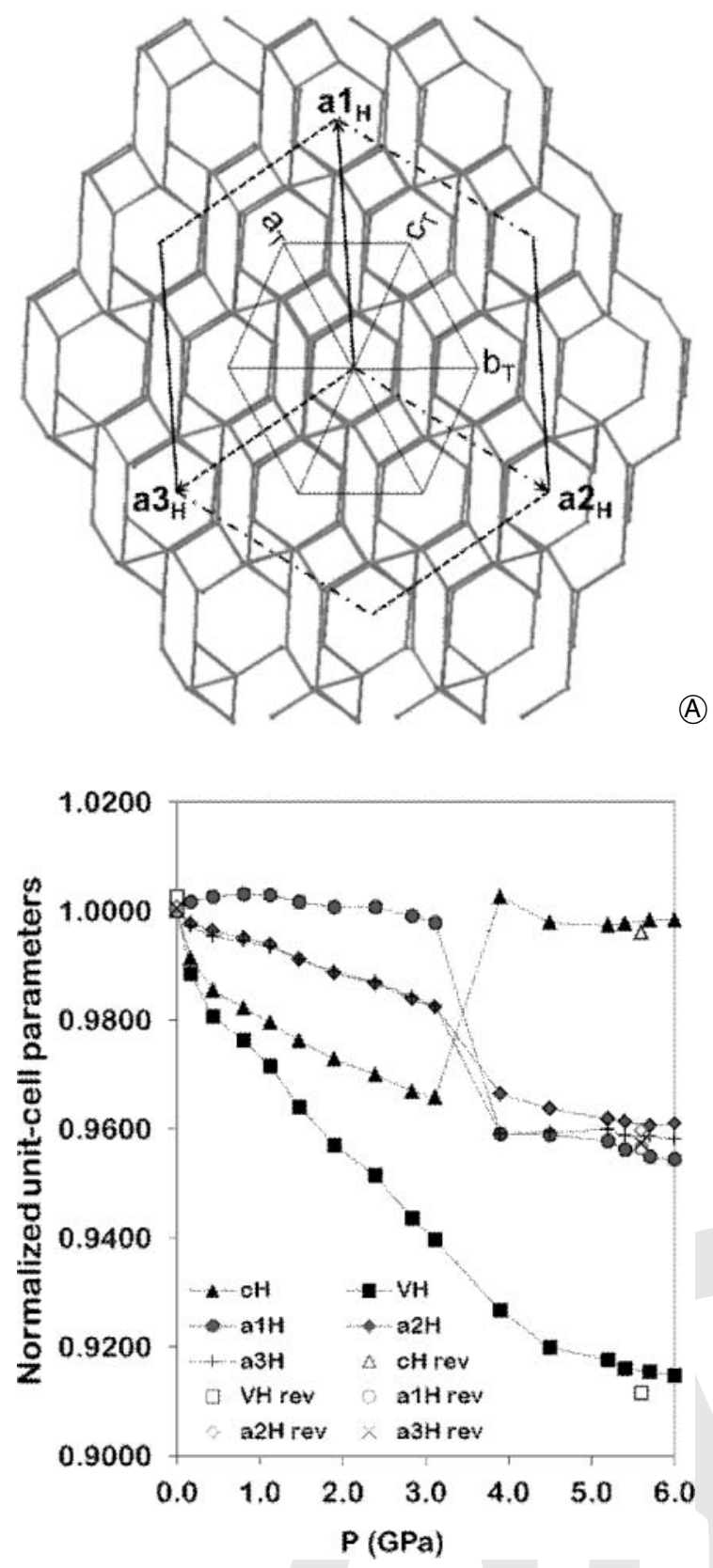

(B)

ii) $0.5<P<3.1 \mathrm{GPa}$ : a shortening of all the parameters is evident.

iii) $P>3.1 \mathrm{GPa}: c_{H}$ parameter suddenly increases by about $4 \%$, whereas all $a_{H}$ parameters decrease. In particular, the increase of $c_{H}$ is fully compensate by the decrease of $a 1_{H}(\sim-4 \%)$ and, hence, the cell volume decrease is rather regular.

Acknowledgements. The Swiss-Norwegian beamline (BM01) at the European Synchrotron Radiation Facility is acknowledged for allocation of beamtime and for technical support during the experiments. L. Marchese kindly provided the ALPO-34 sample. G. D. Gatta and an anonymuos reviewer improved the manuscript with their comments.

\section{References}

Altomare, A.; Camalli, M.; Cuocci, C.; Giacovazzo, C.; Moliterni, A.; Rizzi, R.: EXPO2009: structure solution by powder data in direct and reciprocal space. J. Appl. Cryst. 42 (2009) 1197-1202.
Angel, R. J.; Bujak, M.; Zhao, J.; Gatta, G. D.; Jacobsen, S. D.: Effective hydrostatic limits of pressuremedia for high-pressure crystallographic studies. J. Appl. Cryst. 40 (2007) 26-32.

Arletti, R.; Vezzalini, G.; Morsli, A.; Di Renzo, F.; Dmitriev, V.; Quartieri, S.: Elastic behavior of MFI-type zeolites: 1-Compressibility of Na-ZSM-5 in penetrating and non-penetrating media. Micropor. Mesopor. Mater. 142 (2011) 696-707.

Arletti, R.; Quartieri, S.; Vezzalini, G.: Elastic behavior of zeolite boggsite in silicon oil and aqueous medium: A case of high-pressure-induced over-hydration. Am. Miner. 95 (2010) 1247-1256.

Arletti, R.; Ferro, O.; Quartieri, S.; Sani, A.; Tabacchi, G.; Vezzalini, G.: Structural deformation mechanisms of zeolites under pressure. Am. Miner. 88 (2003) 1416-1422.

Birch, F.: Finite Elastic Strain of Cubic Crystals. Phys. Rev. 71 (1947) 809-824.

Bish, D. L.; Ming. D. W.: Natural Zeolites: occurrence, properties, applications, Reviews in Mineralogy and Geochemistry Vol. 45, Mineralogical Society of America (Washington, D.C) 2001.

Chukanov, N. V.; Pekov, I. V.: Heterosilicates with Tetrahedral-Octahedral Frameworks. Micro- and Mesoporous Mineral Phases (Eds. G. Ferraris, S. Merlino), Reviews in Mineralogy and Geochemistry, Vol. 57, p. 105-143. Mineralogical Society of America and Geochemistry (Washington, DC) 2005.

Corma, A.: From Microporous to Mesoporous Molecular Sieve Materials and Their Use in Catalysis. Chem. Rev. 97 (1997) 23732419.

Dent, L. S.; Smith, J. V.: Crystal Structure of Chabazite, a Molecular Sieve. Nature 181 (1958) 1794-1796.

Engelhardt, G.; Michel, D.: High Resolution Solid-State NMR of Silicates and Zeolites. (Eds. John Wiley \& Sons) Chichester, UK, 1987.

Finger, L. W.; Cox, D. E.; Jephcoat, A. P.: A correction for powder diffraction peak asymmetry due to axial divergence. J. Appl. Cryst. 27 (1994) 892-900.

Fois, E.; Gamba, A.; Medici, C.; Tabacchi, G.; Quartieri, S.; Mazzucato, E.; Arletti, R.; Vezzalini, G.; Dmitriev, V.: High pressure deformation mechanism of Li-ABW: Synchrotron XRPD study and ab initio molecular dynamics simulations. Micropor. Mesopor. Mater. 115 (2008) 267-280.

Forman, R. A.; Piermarini, G. J.; Barnett, J. D.; Block, S.: Pressure Measurement Made by the Utilization of Ruby Sharp-Line Luminescence. Science 176 (1972) 284-285.

Gatta, G. D.: Microporous Materials at High Pressure: Are they Really Soft? In: High-Pressure Crystallography: from fundamental phenomena to technological applications (Eds. E. Boldyreva and P. Dera), pp. 481-491. Sprinter Sciences, 2010.

Gatta, G. D.: Does porous mean soft? On the elastic behaviour and structural evolution of zeolites under pressure. Z. Kristallogr. 223 (2008) 160-170.

Gatta, G. D.; Lee, Y.: On the elastic behaviour of zeolite mordenite: A synchrotron powder diffraction study. Phys. Chem. Miner. 32 (2006) 726-732.

Gatta, G. D.; Comodi, P.; Zanazzi, P. F.; Ballaran, T. F.: Anomalous elastic behavior and high-pressure structural evolution of zeolite levyne. Am. Miner. 90 (2005) 645-652.

Hammersley, A. P.; Svensson, S. O.; Hanfland, M.; Fitch, A. N.; Häusermann, D.: Two dimensional detector software: from real detector to idealized image of two-theta scan. High Pressure Res. 14 (1996) 235-248.

Harding, M. M.; Kariuki, B. M.: Microcrystal structure determination of $\mathrm{AlPO}_{4}-\mathrm{CHA}$ using synchrotron radiation. Acta Cryst. C50 (1994) 852-854.

Krivovichev, S.: Topology of Microporous Structure. In: Micro- and Mesoporous Mineral Phases (Eds. G. Ferraris, S. Merlino), Reviews in Mineralogy and Geochemistry, Vol. 57, pp. 17-68. Mineralogical Society of America and Geochemistry (Washington, DC) 2005 .

Larson, A. C.; Von Dreele, R. B.: GSAS-General Structure Analysis System. Report LAUR 86-748, Los Alamos National Laboratory, Los Alamos, New Mexico, 1996.

Leardini, L.; Quartieri, S.; Vezzalini, G.: Compressibility of microporous materials with CHA topology: 1. Natural chabazite and SAPO-34. Micropor. Mesopor. Mater. 127 (2010) 219-227. 
Marchese, L.; Frache, A.; Gianotti, E.; Martra, G.; Causà, M.; Coluccia, S.: ALPO-34 and SAPO-34 synthesized by using morpholine as templating agent. FTIR and FT-Raman studies of the hostguest and guest-guest interactions within the zeolitic framework. Micropor. Mesopor. Mater. 30 (1999) 145-153.

Mao, H. K.; Xu, J.; Bell, P. M.: Calibration of the ruby pressure gauge to $800 \mathrm{kbar}$ under quasi-hydrostatic conditions. J. Geophys. Res. 91 (1986) 4673-4676.

Martucci, A.; Alberto, A.; Cruciani, G.; Frache, A.; Coluccia, S.; Marchese, L.: Combined Single-Crystal X-ray Diffraction and FTIR Study of Morpholinium-Water Molecular Complexes Embedded in a Chabasite. J. Phys. Chem. B107 (2003) 9655-9661.

Mazzi, F.; Galli, E.: The tetrahedral framework of chabazite. Neues Jahrbuch Fur Mineralogie-Monatshefte 10 (1983) 461-480.

Merlino, S.; Galli, E.; Alberti, A.: The crystal structure of levyne. Tschmarks Min. Petr. Mitt. 22 (1975) 117-129.

Miletich, R.; Allan, D. R.; Kush, W. F.: High pressure single crystal techniques. In: High-Temperature and High-Pressure Crystal Chemistry (Eds. R. M. Hazen, R. T. Downs), Reviews in Mineralogy and Geochemistry, Vol. 41, p. 445-519. Mineralogical Society of America and Geochemical Society (Washington, DC) 2000.

Ori, S.; Quartieri, S.; Vezzalini, G.; Dmitriev, V.: Pressure-induced structural deformation and elastic behavior of wairakite. Am. Miner. 93 (2008a) 53-62.
Ori, S.; Quartieri, S.; Vezzalini, G.; Dmitriev, V.: Pressure-induced over-hydration and water ordering in gismondine: a synchrotron powder diffraction study. Am. Miner. 93 (2008b) 13931403.

Quartieri, S.; Montagna, G.; Arletti, R.; Vezzalini, G.: Elastic behavior of MFI-type zeolites: Compressibility of H-ZSM-5 in penetrating and non-penetrating media. J. Solid State Chem. 184 (2011) 1505-1516.

Ragan, D. D.; Clarke, D. R.; Schiferl, D.: Silicone fluid as a highpressure medium in diamond anvil cells. Rev. Sci. Instrum. 67 (1996) 494-496.

Smith, J. V.; Knowles, C. R.; Rinaldi, F. Crystal structures with a chabazite framework. III. Hydrated Ca-chabazite at +20 and $-150^{\circ}$ C. Acta Cryst. 17 (1964) 374-384.

Smith, J. V.; Rinaldi, F.; Glasser, L. S. D.: Crystal structures with a chabazite framework. II. Hydrated Ca-chabazite at room temperature. Acta Cryst. 16 (1963) 45-53.

Thomson, P.; Cox, D. E.; Hastings, J. B.: Rietveld refinement of Debye-Scherrer synchrotron X-ray data from $\mathrm{Al}_{2} \mathrm{O}_{3}$. J. Appl. Cryst. 20 (1987) 79-83.

Toby, B. H.: EXPGUI, a graphical user interface for GSAS. J. Appl. Cryst. 34 (2001) 210-213. 